\title{
Superalgebras, Symplectic Bosons and the Sugawara Construction
}

\author{
P. Goddard^, D. Olive, and G. Waterson \\ Blackett Laboratory, Imperial College, London SW7 2BZ, United Kingdom
}

\begin{abstract}
New representations of affine Lie algebras are constructed using symplectic bosons of the sort that occur naturally in the BRST treatment of fermionic string theories. These representations are shown to have analogous properties to the current algebra representations in terms of free fermion fields, though they do not act in a positive space. In particular, the condition for the Sugawara construction of the Virasoro algebra to equal the free one is the existence of a superalgebra with a quadratic Casimir operator, paralleling the symmetric space theorem for fermionic field constructions. Both results are seen to be particular cases of a more general super-symmetric space theorem, which arises from considering an affinisation of the superalgebras. These algebras are realised in terms of free fermions and symplectic bosons and lead to a superSugawara construction of the Virasoro algebra. The conditions for this to equal a Virasoro algebra obtained from the free fields are provided by the supersymmetric space theorem.
\end{abstract}

\section{Introduction}

The "quark model current algebra construction" provides the simplest way of obtaining representations of affine Kac-Moody algebras. (For a review see [1].) Given an orthogonal representation of a finite-dimensional compact Lie algebra, $g$,

$$
\left[t^{a}, t^{b}\right]=i f_{c}^{a b} t^{c}
$$

described by real antisymmetric matrices, $M^{a}\left(t^{a} \rightarrow i M^{a}\right)$, we can obtain a representation of the untwisted affine algebra, $\hat{g}$, associated with $g$, by introducing fermi fields, $\psi^{j}(z), 1 \leqq j \leqq \operatorname{dim} M$, defined on the unit circle $|z|=1$ in the complex plane and setting

$$
T^{a}(z)=\frac{i}{2} M_{i j}^{a} \psi^{i}(z) \psi^{j}(z)
$$

\footnotetext{
^ Permanent address: DAMTP, University of Cambridge, Silver Street, Cambridge CB3 9EW, $\mathrm{UK}$
} 
The fermi fields can be either all periodic or all antiperiodic and we shall refer to these as the Ramond (R) and Neveu-Schwarz (NS) cases respectively. Thus

$$
\psi^{j}(z)=\sum_{r} \psi_{r}^{j} z^{-r}
$$

where the sum either runs over $r \in \mathbb{Z}$ (R case) or over $r \in \mathbb{Z}+\frac{1}{2}$ (NS case). In either case $T^{a}(z)$ is periodic,

$$
T^{a}(z)=\sum_{n} T_{n}^{a} z^{-n}
$$

the sum being over $n \in \mathbb{Z}$. Canonical anti-commutation relations for $\psi^{j}(z)$,

$$
\left\{\psi_{r}^{i}, \psi_{s}^{j}\right\}=\delta_{r,-s} \delta^{i j}
$$

imply the defining commutation relations for $\hat{g}$,

$$
\left[T_{m}^{a}, T_{n}^{b}\right]=i f_{c}^{a b} T_{m+n}^{c}+k m \delta_{m,-n} \delta^{a b}
$$

with the central charge $k=\kappa / 2$, where

$$
\operatorname{Tr}\left(M^{a} M^{b}\right)=-\kappa \delta^{a b}
$$

We impose the hermiticity conditions $\psi_{r}^{j \dagger}=\psi_{-r}^{j}$ and, in consequence, $T_{n}^{a \dagger}=T_{-n}^{a}$. (The terms involving $k$ and $\kappa$ in (1.6) and (1.7) can be taken to be diagonal, i.e. proportional to $\delta^{a b}$, if $g$ is compact and simple.) From (1.7) it follows that

$$
Q_{M} \operatorname{dim} M=\kappa \operatorname{dim} g,
$$

where $Q_{M}$ is the value of the quadratic Casimir operator $t^{a} t^{a}$ in the representation $M$.

Directly from $\hat{g}$, we can obtain a Virasoro algebra using Sugawara's construction [2] for the energy-momentum tensor in terms of currents,

$$
\mathscr{L}(z)=\sum_{n} \mathscr{L}_{n} z^{-n}=\frac{1}{2 k+Q_{g}} \sum_{a} \times T^{a}(z) T^{a}(z) \stackrel{\times}{\times},
$$

where the crosses denote normal ordering with respect to $T_{n}^{a}$,

$$
\begin{aligned}
\times T_{m}^{a} T_{n \times}^{a \times} & =T_{m}^{a} T_{n}^{a}, \quad \\
& =T_{n}^{a} T_{m}^{a}, \quad m \geqq 0,
\end{aligned}
$$

and $Q_{g}$ denotes the value of the quadratic Casimir operator in the adjoint representation. Then we have the Virasoro algebra,

with

$$
\left[\mathscr{L}_{m}, \mathscr{L}_{n}\right]=(m-n) \mathscr{L}_{m+n}+\frac{c}{12} m\left(m^{2}-1\right) \delta_{m,-n},
$$

$$
\begin{aligned}
c & =\frac{2 k \operatorname{dim} g}{2 k+Q_{g}} \\
& =\frac{Q_{M} \operatorname{dim} M}{\kappa+Q_{g}} .
\end{aligned}
$$


There is also a construction of the Virasoro algebra based on the free fermion energy-momentum tensor,

$$
L^{\psi}(z)=\sum_{n} L_{n}^{\psi} z^{-n}=\frac{1}{2} \sum_{j}: z \frac{d \psi^{j}(z)}{d z} \psi^{j}(z):+\frac{\varepsilon}{16} \operatorname{dim} M,
$$

where $\varepsilon=0$ (NS case) or 1 ( $\mathrm{R}$ case). This satisfies the Virasoro algebra with $c=\frac{1}{2} \operatorname{dim} M$. Here the colons denote fermionic normal ordering,

$$
\begin{aligned}
: b_{r}^{i} b_{s}^{j}: & =b_{r}^{i} b_{s}^{j}, & & r<0 \\
& =\frac{1}{2}\left[b_{r}^{i}, b_{s}^{j}\right], & & r=0 \\
& =-b_{s}^{j} b_{r}^{i}, & & r>0 .
\end{aligned}
$$

The difference

$$
K(z)=\sum_{n} K_{n} z^{-n}=L^{\psi}(z)-\mathscr{L}(z)
$$

also defines a Virasoro algebra [3]. This difference vanishes if

$$
M_{i j}^{a} M_{k l}^{a}+M_{i k}^{a} M_{l j}^{a}+M_{i l}^{a} M_{j k}^{a}=0 .
$$

It was shown in [3] that this condition is equivalent to the single equation

$$
2 Q_{M}=2 k+Q_{g} .
$$

In [4] it was established that a necessary and sufficient condition for this, i.e. for $\mathscr{L}(z)=L^{\psi}(z)$ is that the representation $M$ should be a representation given by the tangent space to a symmetric space. That is, it should be possible to enlarge $g$ to an algebra $g^{\prime}$ by the addition of generators $\tau^{j}, 1 \leqq j \leqq \operatorname{dim} M$, satisfying (with suitable normalisation),

$$
\begin{aligned}
& {\left[t^{a}, \tau^{j}\right]=i M_{k j}^{a} \tau^{k},} \\
& {\left[\tau^{i}, \tau^{j}\right]=i M_{j i}^{a} t^{a} .}
\end{aligned}
$$

One instance where this occurs is for fermions in the adjoint representation of $g$, corresponding to the type II symmetric space $g \times g / g$. In this case it is possible to construct super-Virasoro generators, $G(z)$, out of the same fermi fields [3]. If we take

$$
G(z)=\sum_{r} G_{r} z^{-r}=\frac{i}{\sqrt{18 Q_{g}}} f^{a b c} \psi_{a}(z) \psi_{b}(z) \psi_{c}(z),
$$

then together with $L^{\psi}(z)$ (or $\mathscr{L}(z)$ ), they form the superalgebra given by (1.11) and

$$
\begin{gathered}
{\left[L_{n}, G_{r}\right]=\left(\frac{n}{2}-r\right) G_{n+r},} \\
\left\{G_{r}, G_{s}\right\}=2 L_{r+s}+\frac{c}{3}\left(r^{2}-\frac{1}{4}\right) \delta_{r,-s},
\end{gathered}
$$


where $c=\frac{1}{2} \operatorname{dim} g$ and $L_{n}$ denotes $L_{n}^{\psi}$ or $\mathscr{L}_{n} . G(z)$ has the same periodicity properties (R or NS) as its constituent fermi fields and the indices $r$ and $s$ in (1.20) correspondingly take integer or half integer values.

In this paper we first establish analogous results to $(1.1-18)$ for bosons instead of fermi fields. The usual construction of $\hat{g}$ in terms of boson fields is the vertex operator construction [5], which is transcendental. The one we shall discuss is bilinear in boson fields. Particular cases have been considered before [6,7] but in Sect. 2 of this paper we shall give a more general treatment to parallel that for fermions. The boson fields used must act in a Hilbert space with an indefinite metric. They are associated with a symplectic form, $J^{\alpha \beta}$, in the same way that the fermions we have considered are associated with the symmetric form $\delta^{i j}$. We introduce $2 n$ real boson fields

$$
\xi^{\alpha}(z)=\sum_{s} \xi_{s}^{\alpha} z^{-s}
$$

where again we can take all the fields to be periodic, $s \in \mathbb{Z}$ (R case) or all antiperiodic, $s \in \mathbb{Z}+\frac{1}{2}$ (NS case), subject to the hermiticity condition $\xi_{s}^{\alpha \dagger}=\xi_{-s}^{\alpha}$. They satisfy the commutation relations

$$
\left[\xi_{r}^{\alpha}, \xi_{s}^{\beta}\right]=i J^{\alpha \beta} \delta_{r,-s},
$$

where $J=J^{\alpha \beta}$ is a non-singular real antisymmetric matrix, $J^{t}=-J$. (Without loss of generality, we could choose a basis for the boson fields so that $J$ is block diagonal with $n$ copies of

$$
\left(\begin{array}{rr}
0 & 1 \\
-1 & 0
\end{array}\right)
$$

along the diagonal and zeros elsewhere.) A pair of such boson fields occurs in the system of BRST ghost fields in the conformally covariant discussion of the fermionic emission vertex [8]. We take the fields to act in a space with vacuum satisfying

$$
\xi_{r}^{\alpha}|0\rangle=0, \quad r>0
$$

In the NS case, this vacuum will be unique, but in the $\mathrm{R}$ case there will have to be a continuum of such vacua (naturally isomorphic to the space of quantum states of a particle moving in $n$ dimensions).

Just as we can build representations of $\hat{g}$, for compact $g$, out of real orthogonal representations using fermi fields, so we can form representations of $\hat{g}$ for suitable, possible noncompact, $g$ out of real symplectic representations using boson fields. Having such a representation of $\hat{g}$, we can perform the Sugawara construction and ask under what circumstances this is equal to the free Virasoro algebra we can form from the symplectic boson fields,

$$
L^{\xi}(z)=\frac{i}{2} J_{\alpha \beta}: z \frac{d \xi^{\alpha}(z)}{d z} \xi^{\beta}(z):-\frac{\varepsilon}{8} n,
$$

where, again, $\varepsilon=0$ (NS case) or 1 (R case),

$$
J_{\alpha \beta} J^{\alpha \delta}=\delta_{\beta}^{\gamma},
$$


and normal ordering is defined analogously to (1.14),

$$
\begin{aligned}
: \xi_{r}^{\alpha} \xi_{s}^{\beta}: & =\xi_{r}^{\alpha} \xi_{s}^{\beta}, & & r<0 \\
& =\frac{1}{2}\left\{\xi_{r}^{\alpha}, \xi_{s}^{\beta}\right\}, & & r=0 \\
& =\xi_{s}^{\beta} \xi_{r}^{\alpha}, & & r>0 .
\end{aligned}
$$

The analogue of the "symmetric space theorem" (1.18) is a "superalgebra theorem" proved in Sect. 3: $\mathscr{L}(z)=L^{\xi}(z)$ if and only if the representation is that given by the fermionic generators $\left\{s^{\alpha}\right\}$ of a superalgebra, $a$, containing $g$ as its bosonic part and possessing a quadratic Casimir operator,

$$
\eta_{a b} t^{a} t^{b}+i J_{\alpha \beta} s^{\alpha} s^{\beta}
$$

where $\eta_{a b}$ is the symmetric invariant form on $g$, used to construct $\mathscr{L}(z)$. We may not just take $\eta_{a b}=\delta_{a b}$, as in (1.9), because, as shown in Sect. 3, $g$ may have to be noncompact.

The simple superalgebras $[9,10]$ with such a Casimir operator are $s u(m \mid n)$, $o s p(m \mid n), d(2 \mid 1 ; \alpha), g(3)$ and $f(4)$, and for these choices of $a, g$ is $s u(m)$ $\oplus s u(n) \oplus u(1)(m \neq n), s o(m) \oplus s p(n), s o(4) \oplus s p(1), g_{2} \oplus s p(1)$ and $s o(7) \oplus s p(1)$, respectively. (For $s u(n \mid n), g=s u(n) \oplus s u(n)$ ) ) These examples of the "superalgebra theorem" are discussed in Sect. 4. The cases $\operatorname{su}(n \mid n), \operatorname{osp}(2 n+2 \mid n)$ and $d(2 \mid 1 ; \alpha)$ are in a sense critical. In these cases, the Sugawara construction fails because $2 k+Q_{g}$ $=0$, and it is not possible to renormalise

$$
\tilde{\mathscr{L}}(z)=\sum_{n} \tilde{\mathscr{L}}_{n} z^{-n}=\frac{1}{2} \eta_{a b} \stackrel{\times}{\times} T^{a}(z) T^{b}(z) \stackrel{\times}{\times}
$$

so that $\widetilde{\mathscr{L}}_{n}$ satisfy the Virasoro algebra. In fact

$$
\left[\tilde{\mathscr{L}}_{n}, \tilde{\mathscr{L}}_{m}\right]=0
$$

In this way, we obtain a natural construction of representations of $\widehat{s u}(n), \widehat{s o}(2 n+2)$ and $\widehat{s p}(n)$ of critical level $-h_{g}$, where $h_{g}$ is the dual Coxeter number of the Lie algebra concerned.

The Virasoro algebra (1.25) has a central term $c=-n$ so that each of the $2 n$ symplectic bosons contributes $-1 / 2$, exactly the negative of the contribution of a fermi field (1.3). Similarly, in (1.25), the term giving the vacuum expectation value to $L_{0}^{\xi}$ is $-1 / 16$ for each Ramond symplectic boson field, again the reverse of a Ramond fermion contribution.

We can understand these relationships, and much else besides, by extending all of our constructions to superalgebras, or more precisely, superalgebras, $a$, possessing a quadratic Casimir operator (1.28). In Sect. 5 we extend the Sugawara construction to affine superalgebras, $\hat{a}$, of this sort, and in Sect. 6 we discuss the construction of representations of such affine superalgebras using orthosymplectic representations of $a$ together with fermion and symplectic boson fields. This then subsumes the construction of representations of affine Lie algebras, $\hat{g}$, using fermion or symplectic boson fields as special cases. When the fermion and boson fields lie in the adjoint representation of the superalgebra, it is also possible to 
construct super-Virasoro generators from these fields. In Sect. 7 we discuss the equivalence of the super-Sugawara construction of Sect. 5 to the "free" expression for the Virasoro algebra in terms of fermion and symplectic boson fields, $L^{\psi}(z)+L^{\xi}(z)$. The condition for this equivalence is given by a "super-symmetric space theorem," which contains the "symmetric space theorem" and the "superalgebra theorem" as special cases. The complete classification of such supersymmetric spaces does not appear to be fully clarified in the mathematics literature as yet, although it has been done for "type I" superalgebras [11,12]. Consequently, we limit ourselves to discussing a few illustrative examples of the super-symmetric space theorem in Sect. 8. Conclusions are given in Sect. 9 and possible developments are discussed.

\section{Symplectic Representations}

We consider $2 n$ symplectic boson fields $\xi^{\alpha}(z), 1 \leqq \alpha \leqq 2 n$, defined by the expansion (1.21) and commutation relations (1.22) acting in a space generated from a vacuum satisfying (1.24). With normal ordering defined by (1.27), we have the operator product relation,

$$
\xi^{\alpha}(z) \xi^{\beta}(\zeta)=: \xi^{\alpha}(z) \xi^{\beta}(\zeta):+i J^{\alpha \beta} \Delta(z, \zeta), \quad|z|>|\zeta|
$$

with the propagator function

$$
\begin{aligned}
\Delta(z, \zeta) & =\frac{z+\zeta}{2(z-\zeta)}, & & \text { periodic }(\mathrm{R}) \text { case } \\
& =\frac{\sqrt{z \zeta}}{z-\zeta}, & & \text { antiperiodic (NS) case } .
\end{aligned}
$$

From these equations it is possible to deduce that $L_{n}^{\xi}$ defined by

$$
L^{\xi}(z)=\sum_{n} L_{n}^{\xi} z^{-n}=\frac{i}{2} J_{\alpha \beta}: z \frac{d \xi^{\alpha}(z)}{d z} \xi^{\beta}(z):-\frac{\varepsilon}{8} n
$$

satisfies the Virasoro algebra with $c=-n$.

Now suppose we have a real symplectic representation of a (possibly noncompact) Lie algebra, $g$, specified by commutation relations (1:1). By this we mean a map $t^{a} \rightarrow i N^{a}$, where $\left(N^{a}\right)_{\beta}^{\alpha} \in \mathbb{R}$,

$$
\left[N^{a}, N^{b}\right]=f_{c}^{a b} N^{c}
$$

and the matrices $N^{a}$ preserve the symplectic form $J=(J)_{\alpha \beta}$. This last statement is equivalent to

$$
J N^{a}=-\left(N^{a}\right)^{t} J=\left(J N^{a}\right)^{t}
$$

that is $R^{a}=J N^{a}$ is a symmetric matrix. We can use $N^{a}$ to obtain a representation of $\hat{g}$ by setting,

$$
T^{a}(z)=\sum_{n} T_{n}^{a} z^{-n}=-\frac{1}{2} R_{\alpha \beta}^{a}: \xi^{\alpha}(z) \xi^{\beta}(z): .
$$


The argument to show that the $T_{n}^{a}$ defined by (2.6) satisfy the algebra $\hat{g}$ parallels the contour deformation argument, reviewed in Subsect. 5.2 of [1], used to establish the corresponding result for the current algebra representation in terms of fermion fields. From (2.1) it follows that

$$
\begin{aligned}
T^{a}(z) T^{b}(\zeta)= & : T^{a}(z) T^{b}(\zeta):-i\left(J N^{a} N^{b}\right)_{\alpha \beta}: \xi^{\alpha}(z) \xi^{\beta}(\zeta): \Delta(z, \zeta) \\
& +\frac{1}{2} \operatorname{Tr}\left(N^{a} N^{b}\right) \Delta(z, \zeta)^{2} .
\end{aligned}
$$

Since

$$
\left(J N^{a} N^{b}\right)_{\alpha \beta}: \xi^{\alpha}(z) \xi^{\beta}(\zeta):=\frac{1}{2}\left(J\left[N^{a}, N^{b}\right]\right)_{\alpha \beta}: \xi^{\alpha}(z) \xi^{\beta}(\zeta):
$$

the usual arguments lead to

$$
\left[T_{m}^{a}, T_{n}^{b}\right]=i f_{c}^{a b} T_{m+n}^{c}+\frac{\kappa}{2} m \eta^{a b} \delta_{m,-n},
$$

where

$$
\operatorname{Tr}\left(N^{a} N^{b}\right)=\kappa \eta^{a b}
$$

Equation (2.10) does not provide as explicit a normalisation as Eq. (1.7) did in the fermionic case. We have replaced $\delta^{a b}$ by the less specific $\eta^{a b}$ to cater to the fact that we shall be considering possibly non-compact algebras. If $g$ is simple, we can fix the normalisation of $\eta^{a b}$ by requiring it to have eigenvalues \pm 1 and to be (equivalent to) $\delta^{a b}$ on the maximal compact subalgebra of $g$. The change of sign between (1.7) and (2.10) ensures the central charge, $k$, of $\hat{g}$ is given by $\kappa / 2$ for both fermion and symplectic boson constructions, and will be seen to be entirely natural in the context of the more general supersymmetric construction given in Sect. 6. In any case the level of the representation (2.9) is given by

$$
x=2 k / \psi^{2}=\kappa / \psi^{2},
$$

where $\psi^{2}$ is the squared length of a long root of $g$, calculated using the metric $\eta_{a b}$ with

$$
\eta_{a b} \eta^{a c}=\delta_{b}^{c}
$$

and is independent of the choice of normalisation.

Whether or not $g$ is simple, we can consider the quadratic Casimir operator $-\eta_{a b} N^{a} N^{b}$, which will have to be a multiple of the identity if $N$ is irreducible,

$$
-\eta_{a b} N^{a} N^{b}=Q_{N} \mathbb{1}_{2 n} .
$$

From (2.10) and (2.13), it follows that

$$
\kappa \operatorname{dim} g=-Q_{N} \operatorname{dim} N,
$$

paralleling (1.8) but with a sign difference we shall understand better in Sect. 6 .

\section{The Sugawara Construction and a Superalgebra Theorem}

We now consider the application of the Sugawara construction to the symplectic boson representation of $\hat{g}$ discussed in Sect. 2 and the circumstances under which 
the Virasoro algebra it produces equals the "free" Virasoro algebra (2.3). We define

$$
\mathscr{L}(z)=\sum_{n} \mathscr{L}_{n} z^{-n}=\frac{1}{\kappa+Q_{g}} \eta_{a b} \times T^{a}(z) T^{b}(z) \stackrel{\times}{\times},
$$

where $Q_{g}$ denotes the value of the quadratic Casimir operator for $g$ in the adjoint representation, with the normalisation implied by $\eta$,

$$
f_{d}^{a c} f_{c}^{b d}=-Q_{g} \eta^{a b}
$$

(for the moment we shall assume $g$ to be simple) and the crosses denote normal ordering as defined in (1.10). Then it follows directly from the algebra (2.9) that the Virasoro algebra (1.11) holds with

$$
c=\frac{\kappa \operatorname{dim} g}{\kappa+Q_{g}}=\frac{x \operatorname{dim} g}{x+h_{g}}
$$

where $h_{g}=Q_{g} / \psi^{2}$ is the dual Coxeter number of $g$. Note that we are assuming implicitly that $\kappa \neq-Q_{g}$ or, equivalently, that the level $x$ of our representation is different from the critical value of $-h_{g}[13]$.

To compare (3.1) and (2.3), we can again follow the arguments used in the fermionic case (see e.g. Subsect. 5.4 of [1]). We can obtain two expressions for $T^{a}(z) T_{a}(\zeta)$ by normal ordering in two ways :

$$
\begin{aligned}
T^{a}(z) T_{a}(\zeta)= & \stackrel{\times}{\times} T^{a}(z) T_{a}(\zeta) \stackrel{\times}{\times}+\frac{\kappa}{2} \operatorname{dim} g \frac{z \zeta}{(z-\zeta)^{2}} \\
= & : T^{a}(z) T_{a}(\zeta):+i Q_{N} J_{\alpha \beta}: \xi^{\alpha}(z) \xi^{\beta}(\zeta): \Delta(z, \zeta) \\
& +\frac{\kappa}{2} \operatorname{dim} g \Delta(z, \zeta)^{2},
\end{aligned}
$$

where (3.4a) has been derivēd exactly as in [1], and (3.4b) follows from (2.7). (We are using $\eta_{a b}, \eta^{a b}$ to lower and raise indices on $T^{a}$ etc.) Now

$$
\Delta(z, \zeta)^{2}-\frac{z \zeta}{(z-\zeta)^{2}}=\frac{\varepsilon}{4}
$$

and from (2.3),

$$
\operatorname{Lim}_{z \rightarrow \zeta}\left\{\frac{i}{2} J_{\alpha \beta}: \xi^{\alpha}(z) \xi^{\beta}(\zeta): \Delta(z, \zeta)\right\}=L^{\xi}(z)+\frac{\varepsilon}{16} \operatorname{dim} N .
$$

Consequently, we can suitably rearrange Eqs. (3.4), using (2.14), and take the limit $z \rightarrow \zeta$ to obtain, just as in the fermionic case,

$$
\frac{1}{2} \times T^{a}(z) T_{a}(z) \stackrel{\times}{\times}=\frac{1}{2}: T^{a}(z) T_{a}(z):+Q_{N} L^{\xi}(z) .
$$

We can see immediately from comparing the values of the central charges of $L^{\xi}(z)$ and $\mathscr{L}(z)$, given by $c=-\frac{1}{2} \operatorname{dim} N$ and (3.3) respectively, that a necessary condition for $L^{\xi}(z)=\mathscr{L}(z)$ is that

$$
2 Q_{N}=\kappa+Q_{g}
$$


Then from (3.1) and (3.7), we see that we require

$$
: T^{a}(z) T_{a}(z):=0 \text {. }
$$

This condition is equivalent to

$$
R_{\alpha \beta}^{a} R_{a \gamma \delta}+R_{\alpha \gamma}^{a} R_{a \delta \beta}+R_{\alpha \delta}^{a} R_{a \beta \gamma}=0
$$

Equation (3.10) is also easily seen to be sufficient for $L^{\xi}(z)=\mathscr{L}(z)$. In the fermionic case, the analogues of (3.8) and (3.10), namely (1.17) and (1.16), were equivalent conditions, but this is no longer the case here because we are dealing with algebras possessing an indefinite invariant form, $\eta_{a b}$. For both fermionic and bosonic constructions, we can construct a third Virasoro algebra, $K(z)=L^{\psi, \xi}(z)-\mathscr{L}(z)$, where $\mathscr{L}(z)$ is given by (1.9) or (3.1) respectively, which has vanishing central charge. When $K(z)$ acts on a positive space, it can be shown [14] that only the trivial representation is possible, i.e. $K(z)$ can be consistently taken to vanish. However, for the symplectic bosons, we no longer have the positive space necessary for this argument to hold and equality of $c$-values for $\mathscr{L}(z)$ and $L^{\xi}(z)$ by itself no longer guarantees equivalence.

Just as (1.16) is equivalent to the statement that the fermionic representation is one given by the tangent space to a symmetric space [4], so (3.10) is equivalent to the statement that $N^{a}$ is given by the fermionic generators of a superalgebra, $a$, but a superalgebra with the special property that it possesses a quadratic Casimir operator. To be more explicit, if Eqs. (2.4), (2.5), and (3.10) hold, then

$$
\begin{gathered}
{\left[t^{a}, t^{b}\right]=i f_{c}^{a b} t^{c},} \\
{\left[t^{a}, s_{\alpha}\right]=i N^{a \beta}{ }_{\alpha} s_{\beta},} \\
\left\{s_{\alpha}, s_{\beta}\right\}=R_{\alpha \beta}^{a} \eta_{a b} t^{b},
\end{gathered}
$$

define a superalgebra, $a$, with quadratic Casimir operator

$$
Q_{a}=\eta_{a b} t^{a} t^{b}+i J^{\alpha \beta} s_{\alpha} s_{\beta} .
$$

(In checking this it is useful to note that the Jacobi identity for $f_{c}^{a b}$ implies the total antisymmetry of $f^{a b c}=f_{d}^{a b} \eta^{d c}$.) Conversely, given a superalgebra (3.11) with quadratic Casimir operator (3.12), it follows from the Jacobi identities that $N^{a}$ defines a representation of $g$, the bosonic part of $a$, and that (3.10) holds; the symmetry of $R^{a}=J N^{a}$ follows from the fact that $Q_{a}$ commutes with $a$. Thus the cases in which $\mathscr{L}(z)=L^{\xi}(z)$ are in one-to-one correspondence with the superalgebras (3.11) possessing Casimir operators (3.12).

For cases where this equivalence holds, $g$ has to be noncompact, or at least possess an indefinite invariant form $\eta_{a b}$ ( $g$ may be the direct sum of compact algebras with the sign of the invariant form of some of the factors taken negative). To show this, define

$$
X^{a}=R_{\alpha \beta}^{a} x^{\alpha} x^{\beta}
$$

and multiply (3.10) by $x^{\alpha} x^{\beta} x^{\gamma} x^{\delta}$ to obtain $3 \eta_{a b} X^{a} X^{b}=0$. If $\eta_{a b}$ were positive (or negative) definite this would imply $X^{a}=0$ for all $x^{\alpha}, x^{\beta}$, and hence $R_{\alpha \beta}^{a}=0$, which is not the case. Thus we conclude that $\eta_{a b}$ has to be an indefinite form. 
In order to fully establish these results, we ought to explicitly allow for the possibility that $g$ is not simple, because this is typically the case for the bosonic part of a superalgebra. Let us suppose that $g$ is the direct product of a semisimple algebra and an abelian algebra (i.e. that it is reductive),

$$
g=\underset{A}{\oplus} g^{A}
$$

where each $g^{A}$ is either simple or a $u(1)$ factor. The invariant symmetric form, $\eta_{a b}$, we use in (3.1) is no longer unique up to an overall constant, but rather has one free parameter for each factor $g^{A}$ of $g$. Equation (3.2) now has to be modified to

$$
f_{d}^{a c} f_{c}^{b d}=-Q_{g}^{A} \eta^{a b},
$$

where we sum the structure constants over each factor $g^{A}$ separately. (They do not in any case connect different factors.) Correspondingly,

and

$$
\begin{aligned}
& \operatorname{Tr}\left(N^{a} N^{b}\right)=\kappa^{A} \eta^{a b}, \\
& -\eta_{a b} N^{a} N^{b}=Q_{N}^{A} 1_{2 n},
\end{aligned}
$$

$$
\kappa^{A} \operatorname{dim} g^{A}=-Q_{N}^{A} \operatorname{dim} N,
$$

the sums over $a, b$ in (3.16b) being over those values corresponding to $g^{A}$. It then follows that we can establish (3.7) more generally with

$$
Q_{N}=\sum_{A} Q_{N}^{A}
$$

If the superalgebra (3.11) exists and the $\eta_{a b}$ we have chosen is one for which we have a Casimir operator (3.12), then evaluating it in the adjoint representation of $a$ shows that

$$
2 Q_{N}=\kappa^{A}+Q_{g}^{A}
$$

for each $A$ as will be seen in Sect. 6. (Here we are assuming that the Casimir operator takes a unique value in the adjoint representation, but if this is not so we can write $a$ as a direct sum of superalgebras, corresponding to different eigenspaces, and consider these separately.) Thus the quantity by which we divide in (3.1) to construct $\mathscr{L}(z)$, is common to the different factors $g^{A}$ and the argument goes through as before to show $\mathscr{L}(z)=L^{\xi}(z)$. Conversely, if these Virasoro algebras are the same, $\kappa^{A}+Q_{g}^{A}$ must have a common value, that is we must choose the parameters in $\eta$ so this is so, in order that (3.1) makes sense. Then (3.18) has to hold and the argument proceeds as before. Hence the "superalgebra theorem" has been established independently of whether or not $g$ is simple. Note that although $\eta$ has a number of free parameters, a priori, their relative size is fixed by the requirement that (3.12) commutes with the superalgebra $a$. We shall see how this works in practice when we illustrate the result by specific examples in the next section.

\section{Examples of the Superalgebra Theorem and Critical Levels}

It is possible to list the simple superalgebras (i.e. those with no non-trivial ideals) possessing quadratic Casimir operators $[9,10]$. They are $s u(p \mid q), \operatorname{osp}(p \mid q), g(3)$, $f(4)$, and $d(2 \mid 1 ; \alpha)$. For each of these, the fermionic generators will provide a 
symplectic representation of the bosonic part of $a$ as in (3.11) and the corresponding symplectic boson field representation of $\hat{g}$ will be such that the Sugawara construction of the Virasoro algebra is equivalent to the free symplectic boson construction. It would be good if we could conclude that all cases in which this happens are direct sums of the cases on this list but, for one thing, the structure of superalgebras in terms of simple superalgebras is more complicated than it is for (compact) Lie algebras [9]. So we are not sure that our list of examples is in any sense complete.

To illustrate the way the theorem works, we shall calculate the form $\eta_{a b}$ in each case and determine the various quantities in Eq. (3.18). To define $\eta_{a b}$, we introduce invariant forms $\bar{\eta}_{a b}^{A}$, which we take to have eigenvalues \pm 1 , again being equal to $\delta_{a b}$ on the maximal compact subgroup of each factor $g^{A}$. We fix the normalisation of the generators so that, for $g^{A}$,

$$
f_{d}^{a c} f_{c}^{b d}=-h_{g^{A}} \bar{\eta}^{a b}
$$

where $h_{g^{A}}$ is the dual Coxeter number of $g^{A}$. Then, restricted to $g^{A}$,

$$
\eta_{a b}=\lambda^{A} \bar{\eta}_{a b}
$$

where $h_{g^{A}}=\lambda^{A} Q_{g}^{A}$ and the level of the representation of $g^{A}$ provided by $N$ is

$$
x^{A}=\lambda^{A} \kappa^{A} .
$$

So, using (3.16-18),

where

$$
\left(x^{A}+h_{g^{A}}\right) / \lambda^{A}=2 \sum_{B} Q_{N}^{B},
$$

$$
Q_{N}^{B}=-\frac{x^{B} \operatorname{dim} g^{B}}{\lambda^{B} \operatorname{dim} N}
$$

If $f$ is the number of factors $g^{A}$ of $g$, Eqs. (4.4-5) constitute $f$ homogeneous equations in the $f$ parameters $\lambda^{B}$. Thus they constitute a non-trivial constraint.

We consider the cases in turn:

(a) $s u(p \mid q), p \neq q: \quad g=s u(p) \oplus s u(q) \oplus u(1), \operatorname{dim} N=2 p q$,

$$
\begin{array}{llll}
h_{s u(p)}=p, & x^{1}=-\frac{1}{2} \cdot 2 q, & \lambda^{1}=1, & Q_{N}^{1}=q\left(p^{2}-1\right) / 2 p q, \\
h_{s u(q)}=q, & x^{2}=-\frac{1}{2} \cdot 2 p, & \lambda^{2}=-1, & Q_{N}^{2}=-p\left(q^{2}-1\right) / 2 p q, \\
h_{u(1)}=0, & x^{3}=\mu(p-q), & \lambda^{3}=\mu, & Q_{N}^{3}=(q-p) / 2 p q ;
\end{array}
$$

(b) $s u(p \mid p): \quad g=s u(p) \oplus s u(p), \operatorname{dim} N=2 p^{2}$,

$$
\begin{array}{llll}
h_{s u(p)}=p, & x^{1}=-\frac{1}{2} \cdot 2 p, & \lambda^{1}=1, & Q_{N}^{1}=\left(p^{2}-1\right) / 2 p ， \\
h_{s u(p)}=p, & x^{2}=-\frac{1}{2} \cdot 2 p, & \lambda^{2}=-1, & Q_{N}^{2}=-\left(p^{2}-1\right) / 2 p ；
\end{array}
$$

(c) $\operatorname{osp}(p \mid q): \quad g=s o(p) \oplus s p(q), \operatorname{dim} N=2 p q$,

$$
\begin{array}{llll}
h_{s o(p)}=p-2, & x^{1}=-2 q, & \lambda^{1}=1, & Q_{N}^{1}=\frac{1}{2}(p-1), \\
h_{s p(q)}=q+1, & x^{2}=-\frac{1}{2} p, & \lambda^{2}=-\frac{1}{2}, & Q_{N}^{2}=-q-\frac{1}{2} ;
\end{array}
$$


(d) $g(3): \quad g=g_{2} \oplus s p(1), \operatorname{dim} N=14$,

$$
\begin{array}{llll}
h_{g_{2}}=4, & x^{1}=1 \cdot(-2), & \lambda^{1}=4, & Q_{N}^{1}=\frac{1}{2}, \\
h_{s p(1)}=2, & x^{2}=-\frac{1}{2} \cdot 7, & \lambda^{2}=-3, & Q_{N}^{2}=-\frac{1}{4} ;
\end{array}
$$

(e) $f(4): \quad g=s o(7) \oplus \operatorname{sp}(1), \operatorname{dim} N=16$,

$$
\begin{aligned}
& h_{\text {so }(7)}=5, \quad x^{1}=1 \cdot(-2), \quad \lambda^{1}=3, \quad Q_{N}^{1}=\frac{7}{8}, \\
& h_{s p(1)}=2, \quad x^{2}=-\frac{1}{2} \cdot 8, \quad \lambda^{2}=-2, \quad Q_{N}^{2}=-\frac{3}{8} ;
\end{aligned}
$$

$$
\begin{array}{llll}
h_{s u(2)}=2, & x^{1}=1 \cdot(-2), & \lambda^{1}=\frac{1}{\mu}, & Q_{N}^{1}=\frac{3}{4} \mu, \\
h_{s u(2)}=2, & x^{2}=1 \cdot(-2), & \lambda^{2}=\frac{1}{1-\mu}, & Q_{N}^{2}=\frac{3}{4}(1-\mu), \\
h_{s p(1)}=2, & x^{3}=-\frac{1}{2} \cdot 4, & \lambda^{3}=-1, & Q_{N}^{3}=-\frac{3}{4} .
\end{array}
$$

(In cases (a) and (f), $\mu$ is an arbitrary constant.)

The cases $s u(p \mid p), \operatorname{osp}(2 q+2 \mid q)$, and $d(2 \mid 1 ; \alpha)$ deserve special attention because for them $Q_{N}=0$, and so the level of the representation of each factor $g^{A}$ is equal to the critical value $-h_{g^{A}}$. In this case, as we mentioned in Sect. 1 , it is not possible to renormalise (1.29) so that we obtain an $\mathscr{L}(z)$ satisfying the Virasoro algebra, but instead the "unrenormalised" operators

$$
\tilde{\mathscr{L}}_{n}^{A}=\frac{1}{2} \eta_{a b} \stackrel{\times}{\times} T^{a}(z) T^{b}(z) \stackrel{\times}{\times},
$$

defined by summing over the indices $a, b$ corresponding to a given factor $g^{A}$, commute:

$$
\left[\tilde{\mathscr{L}}_{m}^{A}, \tilde{\mathscr{L}}_{n}^{B}\right]=0
$$

and, from (3.7), we have instead of $L_{n}^{\xi}=\mathscr{L}_{n}$,

$$
\sum_{A} \tilde{\mathscr{L}}_{n}^{A}=0
$$

\section{The Super-Sugawara Construction}

To see the symmetric space theorem of [4] and the superalgebra theorem of Sect. 3 as particular cases of a more general result, we need to work in the context of affine superalgebras, in particular those obtained by affinisation of the superalgebras that occurred naturally in the results of Sect. 3. Associated with the superalgebra, $a$, of (3.11), with quadratic Casimir operator $Q_{a}$ as in (3.12), we have an affine superalgebra, $\hat{a}$, defined by the relations

$$
\begin{aligned}
& {\left[T_{m}^{a}, T_{n}^{b}\right]=i f_{c}^{a b} T_{m+n}^{c}+k m \eta^{a b} \delta_{m,-n},} \\
& {\left[T_{m}^{a}, S_{r}^{\alpha}\right]=i N_{\beta}^{a \alpha} S_{m+r}^{\beta}} \\
& \left\{S_{r}^{\alpha}, S_{s}^{\beta}\right\}=R_{a}^{\alpha \beta} T_{r+s}^{a}+i k r J^{\alpha \beta} \delta_{r,-s},
\end{aligned}
$$


where $m, n \in \mathbb{Z}$ and either $r, s \in \mathbb{Z}$ (R case) or $r, s \in \mathbb{Z}+\frac{1}{2}$ (NS case), and we have raised and lowered certain indices according to

$$
N_{\beta}^{a \alpha}=J_{\beta \gamma} J^{\alpha \delta} N_{\delta}^{a \gamma}, \quad R_{a}^{\alpha \beta}=J^{\alpha \gamma} N_{\gamma}^{b \beta} \eta_{a b}
$$

for notational convenience. As usual $k$ is a central element; the relative normalisations of its occurrences in (5.1a) and (5.1c) are dictated by the Jacobi identities.

As we explained in Sect. 3, we might as well assume that the quadratic Casimir operator for $a$,

$$
Q_{a}=\eta_{a b} t^{a} t^{b}+i J_{\alpha \beta} s^{\alpha} s^{\beta}
$$

takes a unique value, $2 Q_{N}$, in the adjoint representation of $a$ becuase $a$ can always be written as the direct sum of the superalgebras on which $Q_{a}$ takes different values.

The first result we wish to extend from affine algebras to affine superalgebras is the Sugawara construction; this section is devoted to this objective. The existence of the quadratic Casimir operator (5.3) is, of course, crucial for this. Proceeding as in the familiar affine algebra case, we first define "unrenormalised" Virasoro generators by

$$
\tilde{\mathscr{L}}_{n}=\tilde{\mathscr{L}}_{n}^{T}+\tilde{\mathscr{L}}_{n}^{S}
$$

where

$$
\tilde{\mathscr{L}}^{T}(z)=\sum_{n} \tilde{\mathscr{L}}_{n}^{T} z^{-n}=\frac{1}{2} \eta_{a b} \times T^{a}(z) T^{b}(z) \stackrel{\times}{\times},
$$

and

$$
\tilde{\mathscr{L}}^{S}(z)=\sum_{n} \tilde{\mathscr{L}}_{n}^{S} z^{-n}=\frac{i}{2} J_{\alpha \beta} \times S^{\alpha}(z) S^{\beta}(z) \times \frac{\times}{\times}+\bar{\varepsilon},
$$

where the normal ordering implied by the crosses in $(5.5 b)$ is defined in an exactly analogous way to (1.10) and $\bar{\varepsilon}=0$ or $-k \operatorname{dim} N / 16$ according to whether $S^{\alpha}(z)$ is of $R$ or NS form respectively.

It is easiest to calculate the algebra of the $\tilde{\mathscr{L}}_{n}$ in stages. The usual construction for $\hat{g}$ implies that

$$
\left[\tilde{\mathscr{L}}_{m}^{T}, T_{n}^{a}\right]=-n\left(k+\frac{1}{2} Q_{g}^{A}\right) T_{m+n}^{a}
$$

if $T^{a}$ is a generator of the factor $g^{A}$ of $g$. A similar calculation yields

$$
\left[\tilde{\mathscr{L}}_{m}^{T}, S_{r}^{\alpha}\right]=-\frac{1}{2} r Q_{N} S_{m+r}^{\alpha},
$$

where we have used (2.13). The corresponding results for $\tilde{\mathscr{L}}_{m}^{S}$, obtained in the same way, are

and

$$
\left[\tilde{\mathscr{L}}_{m}^{S}, T_{n}^{a}\right]=-\frac{1}{2} n \kappa^{A} T_{m+n}^{a}
$$

$$
\left[\tilde{\mathscr{L}}_{m}^{S}, S_{r}^{\alpha}\right]=-r\left(k+\frac{1}{2} Q_{N}\right) S_{m+r}^{\alpha} .
$$

Putting (5.6) and (5.7) together and using (3.18), we see that we get a single number $k+Q_{N}$ by which we need to renormalise $\widetilde{\mathscr{L}}_{n}$ :

$$
\begin{gathered}
{\left[\tilde{\mathscr{L}}_{m}, T_{n}^{a}\right]=-n\left(k+Q_{N}\right) T_{m+n}^{a},} \\
{\left[\tilde{\mathscr{L}}_{m}, S_{r}^{\alpha}\right]=-r\left(k+Q_{N}\right) S_{m+r}^{\alpha} .}
\end{gathered}
$$


So we define

$$
\mathscr{L}_{n}=\frac{1}{k+Q_{N}} \tilde{\mathscr{L}}_{n},
$$

and we can then verify that $\mathscr{L}_{n}$ satisfies the Virasoro algebra with

$$
\begin{aligned}
c & =\frac{k}{k+Q_{N}}(\operatorname{dim} g-\operatorname{dim} N) \\
& =\frac{2 k \operatorname{sdim} a}{2 k+Q_{a}},
\end{aligned}
$$

where the superdimension, $\operatorname{sdim} a=\operatorname{dim} g-\operatorname{dim} N$, is defined in the usual way, and $Q_{a}=2 Q_{N}$ is the value of the quadratic Casimir of $a$ in the adjoint representation as will be seen in the next section. This constitutes a very natural generalisation of (1.12a), and we shall see that other formulae generalise similarly. Again we can have critical representations for which $2 k+Q_{a}=0$, where it is not possible to renormalise the Virasoro generators (5.4); examples of this will be given in Sect. 8.

\section{Representations of Affine Superalgebras}

In this section we describe the construction of representations of the affine superalgebra, $\hat{a}$, defined by (5.1). This construction, in terms of fermion and symplectic boson fields, generalises the fermionic current algebra construction of representations of an affine Lie algebra, $\hat{g}$. Just as in that case we start with a real orthogonal representation, as in (1.2), we now start with an orthosymplectic representation of $a$. The new construction will also subsume the symplectic boson construction of Sect. 2. Particular cases of it occur in [6].

By an orthosymplectic representation of $a$, we mean a superalgebra homomorphism $a \rightarrow o s p(m \mid n)$, or, more precisely, osp $\left(m_{1}, m_{2} \mid n\right)$ because the bosonic part, $g$, could have any signature, i.e. $s o\left(m_{1}, m_{2}\right) \oplus s p(n), m_{1}+m_{2}=m$. We can define $\operatorname{osp}\left(m_{1}, m_{2} \mid n\right)$ to be the superalgebra which preserves, in a suitable sense, the form

$$
\tilde{\eta}_{i j} x^{i} x^{j}+i \tilde{J}_{\lambda \mu} \theta^{\lambda} \theta^{\mu}
$$

where $\tilde{\eta}$ is a symmetric $m$-dimensional form of signature $\left(m_{1}, m_{2}\right)$ and $\widetilde{J}$ is a symplectic form of dimension $2 n$. Then a representation of $a$ takes the form

$$
\begin{aligned}
& t^{a} \rightarrow i\left(\frac{M^{a}}{0} \mid \frac{0}{K^{a}}\right)=i \mathbf{M}^{a}, \\
& s^{\alpha} \rightarrow\left(\frac{0}{Y^{\alpha}} \mid \frac{i X^{\alpha}}{0}\right)=i \mathbf{X}^{\alpha},
\end{aligned}
$$

where $M^{a}, K^{a}, X^{\alpha}, Y^{\alpha}$ are real matrices and $i \mathbf{M}^{a}, i \mathbf{X}^{\alpha}$ satisfy (3.11) and also the conditions

$$
\tilde{\eta} M^{a}=-\left(\tilde{\eta} M^{a}\right)^{t}, \quad \tilde{J} K^{a}=\left(\tilde{J} K^{a}\right)^{t}, \quad \tilde{\eta} X^{\alpha}=\left(Y^{\alpha}\right)^{t} \tilde{J}
$$


in order that they should preserve (6.1). Then, if we define

$$
\begin{aligned}
T^{a}(z) & =\frac{i}{2} \psi(z)^{t} \tilde{\eta} M^{a} \psi(z)-\frac{1}{2} \xi(z)^{t} \tilde{J} K^{a} \xi(z), \\
S^{\alpha}(z) & =\frac{1}{2} \psi(z)^{t} \tilde{\eta} X^{\alpha} \xi(z)-\frac{1}{2} \xi(z)^{t} \tilde{J} Y^{\alpha} \psi(z) \\
& =\psi(z)^{t} \tilde{\eta} X^{\alpha} \xi(z),
\end{aligned}
$$

where $\psi^{j}(z), 1 \leqq j \leqq m$, and $\xi^{\lambda}(z), 1 \leqq \lambda \leqq 2 n$ are respectively fermion and boson fields satisfying

$$
\left\{\psi_{r}^{i}, \psi_{s}^{j}\right\}=\tilde{\eta}^{i j} \delta_{r,-s}, \quad\left[\xi_{r}^{\lambda}, \xi_{s}^{\mu}\right]=i \tilde{J}^{\lambda \mu} \delta_{r,-s},
$$

we obtain a representation of $\hat{a}$. (Note there is no need to explicitly normal order the expressions in (6.4) and (6.5).) This representation has $k=\kappa / 2$, where now

$$
\begin{gathered}
\operatorname{Str}\left(\mathbf{M}^{a} \mathbf{M}^{b}\right) \equiv \operatorname{Tr}\left(M^{a} M^{b}\right)-\operatorname{Tr}\left(K^{a} K^{b}\right)=-\kappa \eta^{a b}, \\
\operatorname{Str}\left(\mathbf{X}^{\alpha} \mathbf{X}^{\beta}\right) \equiv i \operatorname{Tr}\left(X^{\beta} Y^{\alpha}\right)-i \operatorname{Tr}\left(X^{\alpha} Y^{\beta}\right)=-2 i \operatorname{Tr}\left(X^{\alpha} Y^{\beta}\right)=-i \kappa J^{\alpha \beta} .
\end{gathered}
$$

(Here Str (M) denotes the supertrace of the matrix $\mathbf{M}$ defined in the conventional way.) To be sure that Eqs. (6.7) will necessarily hold for some $\kappa$, we need that (5.3) is the unique quadratic Casimir operator for $a$, up to a scale, and that the representation (6.2) of $a$ is irreducible.

Following familiar arguments, we can relate $\kappa$ to the value of $Q_{\mathbf{M}}$ of the quadratic Casimir operator for $a$ in the representation (6.2) by using

$$
\begin{aligned}
& \eta_{a b} M^{a} M^{b}+J_{\alpha \beta} X^{\alpha} Y^{\beta}=-Q_{\mathbf{M}} \mathbb{1}_{m}, \\
& \eta_{a b} K^{a} K^{b}+J_{\alpha \beta} Y^{\alpha} X^{\beta}=-Q_{\mathbf{M}} \mathbb{1}_{2 n},
\end{aligned}
$$

together with (6.7), to obtain

$$
\kappa \operatorname{sdim} a=Q_{\mathbf{M}} \operatorname{sdim} \mathbf{M},
$$

where $\operatorname{sim} \mathbf{M}=m-2 n$. In the context of the general Eqs. (6.7) and (6.9), the sign difference between (1.7) and (2.10), and between (1.8) and (2.14), respectively, appear natural.

We can take each of the two fields $\psi(z)$ and $\xi(z)$ to be either Ramond or NeveuSchwarz independently. If we make the same choice in each case we obtain the $\mathrm{R}$ form of $\hat{a}$ and if we make different choices we obtain the NS form.

A particular and important case of this construction is provided by the adjoint representation of $a$. This corresponds to taking

$$
M_{j}^{a i}=-f_{j}^{a i}, \quad K_{\mu}^{a \lambda}=-N_{\mu}^{a \lambda}, \quad X_{\lambda}^{\alpha i}=N_{\lambda}^{i \alpha}
$$

and identifying $\eta$ and $\tilde{\eta}, J$ and $\widetilde{J}$. In this case, $\kappa=Q_{\mathbf{M}}=Q_{a}=2 Q_{N}$. The proof of Eq. (3.18) follows from inserting (6.10) into Eqs. $(6.8 \mathrm{a}, \mathrm{b})$.

For a general orthosymplectic representation of $\hat{a}$, we can write the central charge (5.10) as

$$
c=\frac{\kappa \operatorname{sdim} a}{\kappa+Q_{a}}=\frac{Q_{\mathbf{M}} \operatorname{sdim} \mathbf{M}}{\kappa+Q_{a}},
$$


and for the adjoint representation this becomes $\frac{1}{2} \operatorname{sdim} a$, which is also the value of $c$ for $L^{\psi}(z)+L^{\xi}(z)$, where $L^{\psi}(z)$ is given by (1.13) and $L^{\xi}(z)$ by (1.25), consistent with $\mathscr{L}(z)=L^{\psi}(z)+L^{\xi}(z)$ holding for this representation. In the next section we shall see that this is indeed the case for the adjoint representation, as we would expect from previous results, and find the general condition for this identification to obtain.

The adjoint representation is of special interest because in this case it is also possible to construct super-Virasoro generators out of the same fermi and symplectic boson fields, analogous to the result (1.19). We find that by defining

$$
\begin{aligned}
G(z)=\sum_{n} G_{n} z^{-n}= & \frac{1}{\sqrt{Q_{N}}}\left\{\frac{i}{6} f^{a b c} \psi_{a}(z) \psi_{b}(z) \psi_{c}(z)\right. \\
& \left.+\frac{1}{2}\left(J N^{a}\right)_{\alpha \beta} \psi_{a}(z) \xi^{\alpha}(z) \xi^{\beta}(z)\right\},
\end{aligned}
$$

then together with $L(z)=L^{\psi}(z)+L^{\xi}(z)$, these operators obey the superalgebra given by (1.11) and (1.20) with $c=\frac{1}{2} \operatorname{sdim} a . G(z)$ has the same Ramond or Neveu-Schwarz properties as its constituent fermion fields, independent of the periodicity properties of the symplectic bosons.

\section{A Super-Symmetric Space Theorem}

We have seen in the last two sections how results on the Sugawara construction, and on the fermionic and symplectic boson representations of affine Lie algebras fit neatly into a supersymmetric framework. To extend this process, we shall generalise the symmetric space theorem [4] and the superalgebra theorem of Sect. 3 into a super-symmetric space theorem. This will give the conditions under which the super-Sugawara construction of the Virasoro algebra, applied to the orthosymplectic representations of the affine superalgebra, $\hat{a}$, constructed in Sect. 5, equals the sum of the Virasoro algebras associated with the free fermion and symplectic boson fields used in the construction, i.e.

$$
\mathscr{L}(z)=L^{\psi}(z)+L^{\xi}(z)
$$

To state the result, we need to generalise certain concepts from Lie algebras to superalgebras. Firstly, suppose the superalgebra $a=g+s$ is contained in a larger superalgebra $a^{\prime}=g^{\prime}+s^{\prime}$ ( $g$ and $g^{\prime}$ being the bosonic parts of $a$ and $a^{\prime}$ respectively, and $s$ and $s^{\prime}$ being their fermionic parts). Suppose that the quadratic Casimir operator $Q_{a}$ for $a$ extends to one $Q_{a^{\prime}}$ for $a^{\prime}$. Then we can write

$$
g^{\prime}=g+\gamma, \quad s^{\prime}=s+\sigma,
$$

where $\gamma, \sigma$ are orthogonal to $g, s$ respectively, with respect to the quadratic form on $a^{\prime}$ corresponding to $Q_{a^{\prime}}$. It then follows that

$$
\begin{array}{ll}
{[g, \gamma] \subset \gamma,} & {[g, \sigma] \subset \sigma,} \\
{[s, \gamma] \subset \sigma,} & \{s, \sigma\} \subset \gamma,
\end{array}
$$


and so $\gamma+\sigma$ provides a representation space for $a$; this is the representation associated with $a^{\prime} / a$. Secondly, suppose that $a^{\prime}$ has an automorphism, $\varrho$, of order 2 , which commutes with $Q_{a^{\prime}}$, and such that $a$ is the subalgebra on which $\varrho=1$. Then $\gamma+\sigma$ is the subspace on which $\varrho=-1$ and

$$
[\gamma, \gamma] \subset g, \quad[\gamma, \sigma] \subset s, \quad\{\sigma, \sigma\} \subset g .
$$

This is the natural generalisation to superalgebras of the concept of a symmetric space for Lie algebras; we shall call $a^{\prime} / a$ a super-symmetric space if (7.4) holds.

The main result of this section is that (7.1) holds if and only if the orthosymplectic representation of $a$ used is one associated with a super-symmetric space $a^{\prime} / a$.

Generalising (3.7) we have

$$
\tilde{\mathscr{L}}(z)=\frac{1}{2} \eta_{a b}: T^{a}(z) T^{b}(z):+\frac{i}{2} J_{\alpha \beta}: S^{\alpha}(z) S^{\beta}(z):+Q_{\mathbf{M}} L(z)
$$

where $L(z)=L^{\psi}(z)+L^{\xi}(z)$. Again, comparing the value (6.11) of $c$ for $\mathscr{L}(z)$ with the value $\frac{1}{2} \operatorname{sdim} \mathbf{M}$ for $L(z)$, we see that a necessary condition for $\mathscr{L}(z)=L(z)$ is that

$$
2 Q_{\mathbf{M}}=\kappa+Q_{a}
$$

and also, from (7.5) and (5.9), that

$$
\frac{1}{2} \eta_{a b}: T^{a}(z) T^{b}(z):+\frac{i}{2} J_{\alpha \beta}: S^{\alpha}(z) S^{\beta}(z):=0 .
$$

Equation (7.7) by itself is sufficient for $\mathscr{L}(z)=L(z)$ and is equivalent to the conditions

$$
\begin{gathered}
M_{i j}^{a} M_{a k l}+M_{i k}^{a} M_{a l j}+M_{i l}^{a} M_{a j k}=0, \\
K_{\lambda \mu}^{a} K_{a v \varrho}+K_{\lambda \varrho}^{a} K_{a \mu \nu}+K_{\lambda v}^{a} K_{a \varrho \mu}=0, \\
J_{\alpha \beta} X_{\lambda}^{\alpha i} X_{\mu}^{\beta j}+J_{\alpha \beta} X_{\mu}^{\alpha i} X_{\lambda}^{\beta j}-K_{\lambda \mu}^{a} M_{a}^{i j}=0,
\end{gathered}
$$

where $K_{\lambda \mu}^{a}=\widetilde{J}_{\lambda \nu} K^{a v}{ }_{\mu}$, and we use $\eta, \tilde{\eta}$ to raise and lower indices. Equations (7.8) are precisely the conditions that allow us to extend $a$ to a superalgebra $a^{\prime}$, in such a way that $a^{\prime} / a$ is a super-symmetric space, through the addition of generators $\tau^{j}, 1 \leqq j \leqq m$, and $\sigma^{\lambda}, 1 \leqq \lambda \leqq 2 n$ satisfying

$$
\begin{aligned}
& {\left[t^{a}, \tau^{j}\right]=i M_{\mathrm{k}}^{a j} \tau^{\mathrm{k}}, \quad\left[t^{a}, \sigma^{\lambda}\right]=i K_{\mu}^{a \lambda} \sigma^{\mu},} \\
& {\left[\tau^{j}, s^{\alpha}\right]=i X_{\lambda}^{\alpha j} \sigma^{\lambda}, \quad\left\{s^{\alpha}, \sigma^{\lambda}\right\}=\widetilde{J}^{\lambda \mu} X_{j \mu}^{\alpha} \tau^{j},} \\
& {\left[\tau^{i}, \tau^{j}\right]=i M_{a}^{j i} t^{a}, \quad\left\{\sigma^{\lambda}, \sigma^{\mu}\right\}=K_{a}^{\lambda \mu} t^{a},} \\
& {\left[\tau^{j}, \sigma^{\lambda}\right]=i J_{\beta \alpha} \tilde{J}^{\lambda \mu} X^{\beta j}{ }_{\mu} s^{\alpha} .}
\end{aligned}
$$

This completes the proof of our super-symmetric space theorem.

A common feature of symmetric spaces and superalgebras is a $\mathbb{Z}_{2}$ grading, and this is a crucial aspect of their role in the analogy between fermion and symplectic boson representations of affine Lie algebras. A super-symmetric space has a richer $\mathbb{Z}_{2} \times \mathbb{Z}_{2}$ grading (because we can define one involution by reversing the sign of the 
fermionic generators and another by reversing the sign of $\gamma+\sigma$ ). Corresponding to the three $\mathbb{Z}_{2}$ subgroups of $\mathbb{Z}_{2} \times \mathbb{Z}_{2}$, we have three structures: the superalgebra $g+\sigma$, the symmetric space $g^{\prime} / g$ and the superalgebra $a=g+s$. Of these, $g+\sigma$ is associated with (7.8b) and $g^{\prime} / g$ with (7.8a). In consequence, we have that $L^{\psi}(z)$ and $L^{\xi}(z)$ equal the Sugawara constructions for the fermionic and symplectic boson representations of $\hat{g}$ given by $\gamma$ and $\sigma$, respectively, as well as the more complex result (7.1).

The classification of automorphisms of order 2 of simple Lie superalgebras has been given in $[11,12]$. The corresponding super-symmetric spaces are the analogues of type I symmetric spaces, but it is not clear how a general supersymmetric space is related to these. Consequently, we restrict ourselves to a few illustrative examples in the next section.

\section{Examples of the Super-Symmetric Space Theorem}

As was shown in Sect. 6, the super-Sugawara construction of the Virasoro algebra gives a $c$-value equal to the free field value of $\frac{1}{2} \operatorname{sdim} a$ when the fields are taken in the adjoint representation of $a$. Also, the conditions (7.8) are satisfied by the quantities given in (6.10), thus establishing the equality of the two constructions. This is related to a super-symmetric space $a \times a / a$ analogous to the type II symmetric spaces discussed in [4]. The analogues of type I symmetric spaces provide further examples of the super-symmetric space theorem.

As a first example, consider the superalgebra $\operatorname{osp}(m \mid n)$. The construction of the corresponding affine superalgebra from fields in the adjoint representation involves $\frac{1}{2} m(m-1)+n(2 n+1)$ fermions and $2 m n$ symplectic bosons, giving rise to a superSugawara Virasoro algebra with

$$
c=\frac{1}{4}(m-2 n)(m-2 n-1)=\frac{1}{2} \operatorname{sdim} \operatorname{osp}(m \mid n),
$$

as in Sect. 6. Another possibility is to take $m$ fermions and $2 n$ symplectic bosons and construct the first $\frac{1}{2} m(m-1) T^{a}(z)$ generators appearing in (6.4) out of the fermions alone, with the remaining $n(2 n+1)$ generators being constructed from the bosons as in Sect. 2. The $2 \mathrm{~nm} S^{\alpha}(z)$ generators of Eq. (6.5) are formed from all possible bilinear products of the fermion and boson fields. Using (4.3) and the normalisation of roots implied by Sect. 4 , we find the corresponding $T_{n}^{a}, S_{r}^{\alpha}$ obey (5.1) with $k=1 / 2$. Combining this with the value of $Q_{a}=2 Q_{N}=(m-2-2 n)$, we find the superSugawara construction has a $c$-value given by (6.11) of

$$
c=\frac{k \operatorname{sdim} \operatorname{osp}(m \mid n)}{k+Q_{N}}=\frac{1}{2}(m-2 n),
$$

which is the same as the free field value for $m$ fermions and $2 n$ symplectic bosons. This suggests that we may have an equivalence of the two Virasoro algebras, $\mathscr{L}(z)$ and $L(z)$, but the proof requires that we can find an appropriate super-symmetric space. The relevant space in this case turns out to be $\operatorname{osp}(m+1 \mid n) / o s p(m \mid n)$. We can check that this yields the right number of bosons and fermions required for the construction. The number of fermions is given by the difference in the number of $T^{a}(z)$ generators of $a^{\prime}=o s p(m+1 \mid n)$ and $a=o s p(m \mid n)$ (i.e. by $\left.\operatorname{dim} g^{\prime}-\operatorname{dim} g\right)$, which is just $m$. Similarly, the number of symplectic bosons is given by the difference in the 
number of fermionic generators, $S^{\alpha}(z)$, of the two superalgebras, which here is $2 n(m+1)-2 n m=2 n$. In [6], the particular case of $m=1$ was discussed. Note that $k+Q_{N}=0$ when $m=2 n+1$, giving rise to critical representations of $\widehat{o s p}(2 n+1 \mid n)$.

Another, slightly more complicated, example involves the super-symmetric space $s u(m \mid 2 n) / \operatorname{osp}(m \mid n)$. Using the same counting arguments as above, we find this space provides us with $\frac{1}{2}(m-1)(m+2)+(2 n+1)(n-1)+1$ fermions (assuming $m \neq 2 n$ for simplicity) and $2 m n$ symplectic bosons with which to construct an $\widehat{o s p}(m \mid n)$ representation. The $T^{a}(z)$ generators are constructed out of a sum of fermionic and bosonic parts, where the bosonic part involves symplectic bosons in the adjoint representation. The first $\frac{1}{2} m(m-1) T^{a}(z)$ generators have a fermionic part constructed from fermions in a $\frac{1}{2}(m-1)(m+2)$ dimensional representation of so $(m)$, while the remaining $n(2 n+1)$ generators involve fermions in a $(2 n+1)(n-1)$ dimensional representation of $\operatorname{sp}(n)$. The value of $k$ obtained is $\frac{1}{2}(m+2-2 n)$ and the super-Sugawara construction gives

$$
c=\frac{k \operatorname{sdim} o s p(m \mid n)}{k+Q_{N}}=\frac{1}{4}(2 n-m-2)(2 n-m+1),
$$

which again is the same as the value obtained by a free field construction.

As a final example, consider the super-symmetric space $s u(m+1 \mid n) / s u(m \mid n)$ $\times u$ (1) (assuming $n \neq m$ or $m+1$ for simplicity), which provides us with $2 m$ fermions and $2 n$ symplectic bosons. As discussed in [1], when constructing affine Lie algebras from fermions in the $m$ dimensional defining representation of $s u(m)$, it is necessary to double the number of fermions and consider a $2 m$ dimensional real representation. This results in an extra $\hat{u}(1) \mathrm{Kac}-$ Moody algebra, commuting with the $\widehat{s u}(m)$ one and the corresponding $u(1)$ Sugawara construction has to be taken into account when discussing equality with free field constructions. The same thing occurs here, where the first $\left(m^{2}-1\right) T^{a}(z)$ generators are constructed using fermions in a $2 m$ dimensional real representation of $s u(m)$, with the remainder formed from the $2 n$ bosons. The super-Sugawara construction for $\widehat{s u}(m \mid n)$ with $k=1 / 2$ then gives

$$
c=\frac{k \operatorname{sdim} s u(m \mid n)}{k+Q_{N}}=m-n-1 .
$$

This is 1 less than the free field value, but, as noted above, the extra contribution to the super-Sugawara construction of the $u(1)$ factor has to be added to (8.4) and this does indeed contribute $c=1$.

The classification of "type I" super-symmetric spaces is given in $[11,12]$, but the above examples embody the general features.

\section{Conclusions and Discussion}

By using symplectic bosons to construct affine Lie algebras and related Virasoro algebras, we have obtained results analogous to the fermi field constructions given in $[3,4]$. The analogue of the symmetric space theorem is a "superalgebra theorem." We have a complete list of examples of this theorem for simple superalgebras with quadratic Casimir operator given by (3.12) since these have been 
classified in [9]. Unfortunately, it is more complicated to write an arbitrary superalgebra in terms of simple superalgebras than it is to decompose a compact Lie algebra into simple ones. Thus there may be more instances where the theorem applies than those cases obtained by simply taking direct products of the simple superalgebras for which the result holds.

Combining the symplectic boson and fermion constructions led to realisations of affine superalgebras. The starting point for fermionic realisations of affine algebras was an orthogonal representation of the corresponding Lie algebra. Similarly, for the affine superalgebras, the construction involves an orthosymplectic representation of the corresponding superalgebra. Constructing superSugawara Virasoro generators as described in Sect. 5 enabled us to prove a "supersymmetric space theorem," which contains the symmetric space and superalgebra theorems as special cases. Again, although the "type I" super-symmetric spaces have been classified, the complete classification remains to be clarified.

We have defined critical representations of affine Lie algebras to be those representations for which the Sugawara construction fails because the candidate Virasoro generators cannot be renormalised to give the correct algebra. As we found in Sect. 4, in these cases instead of establishing the equivalence of the Sugawara and free field constructions, the superalgebra theorem gave Eq. (4.8), which must be interpreted as meaning $\sum_{A} \tilde{\mathscr{L}}_{n}^{A}$ annihilate highest weight states of the corresponding affine algebra. These are states $|\Psi\rangle$ satisfying

$$
T_{n}^{a}|\Psi\rangle=0, \quad n>0 \text {. }
$$

There are now many extra null states (i.e. states satisfying (9.1) and being orthogonal to any solution of these equations) of the form

$$
\left(\tilde{\mathscr{L}}_{-r}^{A}\right)^{n_{r}} \ldots\left(\tilde{\mathscr{L}}_{-1}^{A}\right)^{n_{1}}|\Psi\rangle
$$

The occurrence of such an unexpectedly large set of such null states is one of the prime characteristics of the "no ghost theorem" [15] of string theory, which suggests we may have an analogous result for affine Lie algebras. The condition for the existence of a critical representation is that the level of the representation should be the negative of the dual Coxeter number of the algebra. The more recent approaches to the "no ghost theorem" involve the construction of a nilpotent BRST charge, which defines an equivalence relation between physical states. However, when these methods are applied to the affine Lie algebra, the nilpotency condition requires the level of the representation to be the negative of $t$ wice the dual Coxeter number [16]. A similar discrepancy of a factor of 2 occurs in comparing the condition for the existence of critical representations of the affine superalgebras with the condition for a corresponding nilpotent BRST charge. These discrepancies have caused certain confusion in the literature [17] and require further investigation.

Another speculative idea is to consider whether vertex operators have a role to play in the framework of symplectic bosons we have established. They do arise in constructing the superconformal ghosts of fermionic string theories [8], which are the simplest examples of the symplectic boson fields we have considered.

It is also interesting to note that as well as the superconformal ghosts of string theory, our formalism also naturally includes the conformal ghosts. This is because 
the fermionic anti-commutation relations given in Eq. (6.6) involve $\tilde{\eta}^{i j}$, rather than $\delta^{i j}$, which can be of indefinite signature. In particular, choosing $i, j=1,2$ and $\tilde{\eta}=\operatorname{diag}(1,-1)$ gives rise to fields obeying the same anti-commutation relations as the conformal ghosts. Both bosonic and fermionic ghosts are thus naturally incorporated into the framework of representations of affine superalgebras discussed in Sect. 6. However, the Virasoro algebras we have discussed are different from the ones normally used in string theory, where derivatives of $u(1)$ ghost currents are added to the Sugawara Virasoro generators in order to give the ghost fields their usual conformal weights. The precise connection between our constructions and those occurring in the ghost sector of string theories remains to be established.

Acknowledgements. We are very grateful to Victor Kac for discussions. Peter Goddard is grateful to the Theoretical Physics Group at Imperial College for hospitality and to the S.E.R.C. for a Visiting Fellowship. Gavin Waterson would like to thank the S.E.R.C. for a Studentship.

\section{References}

1. Goddard, P., Olive, D.: Int. J. Mod. Phys. A 1, 303 (1986)

2. Sugawara, H.: A field theory of currents. Phys. Rev. 170, 1659 (1968)

3. Goddard, P., Olive, D.: Kac-Moody algebras, conformal symmetry and critical exponents. Nucl. Phys. B257 [FS14], 226 (1985)

4. Goddard, P., Nahm, W., Olive, D. : Symmetric spaces, Sugawara's energy momentum tensor in two dimensions and free fermions. Phys. Lett. 160B, 111 (1985)

5. Frenkel, I., Kac, V.: Invent. Math. 62, 23 (1980); Segal, G.: Unitary representations of some infinite dimensional groups. Commun. Math. Phys. 80, 301 (1981)

6. Feingold, A., Frenkel, I.: Adv. Math. 56, 117 (1985)

7. Ezawa, Z.F., Nakamura, S., Tezuka, A.: Tohoku University preprint TU/86/304

8. Friedan, D., Martinec, E., Shenker, S.: Conformal invariance, supersymmetry and string theory. Nucl. Phys. B271, 93 (1986)

9. Kac, V.G.: Adv. Math. 26, 8 (1977)

10. Freund, P.G.O.: Introduction to supersymmetry. Cambridge: Cambridge University Press 1986

11. Serganova, V.V.: Funct. Anal. Appl. 17, 200 (1983) [English translation]

12. Leites, D.A.: Itogi Nauki i Tekhniki, Ser. Sov. Prob. Mat., Noveishie Dostizheniya, 25, 3 (1984) and references therein

13. Kac, V.: In: Geometrical and topological methods in field theory. Singapore: World Scientific 1987

14. Gomes, J.F.: The triviality of representations of the Virasoro algebra with vanishing central element and $L_{0}$ positive. Phys. Lett. 171 B, 75 (1986)

15. Brower, R.C.: Spectrum-generating algebra and no-ghost theorem for the dual model. Phys. Rev. D6, 1655 (1972); Goddard, P., Thorn, C.B.: Compatibility of the dual pomeron with unitarity and the absence of ghosts in the dual resonance model. Phys. Lett. 40 B, 235 (1972)

16. Kawai, T.: Remarks on a class of BRST operators. Phys. Lett. B168, 355 (1986)

17. Altschuler, D.: University of Geneva preprint UGVA-DPT 1986/10-521

Communicated by L. Alvarez-Gaumé 
